\title{
PAPER
}

\section{Relativistic solar sails}

To cite this article: J Güémez and M Fiolhais 2018 Eur. J. Phys. 39035601

View the article online for updates and enhancements.

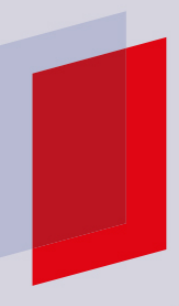

\section{IOP ebooks}

Bringing you innovative digital publishing with leading voices

to create your essential collection of books in STEM research.

Start exploring the collection - download the first chapter of every title for free. 


\title{
Relativistic solar sails
}

\author{
J Güémez ${ }^{1}$ and $M$ Fiolhais ${ }^{2,3}$ (1) \\ ${ }^{1}$ Department of Applied Physics, Faculty of Sciences, University of Cantabria, Av. de \\ Los Castros, E-39005 Santander, Spain \\ ${ }^{2}$ Physics Department and CFisUC, University of Coimbra, 3004-516 Coimbra, \\ Portugal \\ E-mail: guemezj@unican.es and tmanuel@fis.uc.pt
}

Received 23 August 2017, revised 11 December 2017

Accepted for publication 20 December 2017

Published 7 March 2018

\begin{abstract}
We apply the four-vector formalism of special relativity to describe various interaction processes of photons with a solar sail, in two cases: when the sail's surface is a perfect mirror, and when it is a body coated with a totally absorbing material. We stress the pedagogical value of implementing simultaneously both the linear momentum and the energy conservation in a covariant fashion, as our formalism inherently does. It also allows for a straightforward change of the description of a certain process in different inertial reference frames.
\end{abstract}

Keywords: special relativity, radiation pressure, radiation reflection and absorption

\section{Introduction}

The solar sail is an idealised device that has been considered to be used in long outer space journeys [1], such as missions to Mars. It is based on the pressure exerted upon the surface of a large object - the sail-by radiation coming, for instance, from a light source such as the Sun. The idea has already merited the attention of various authors that studied several possibilities, such as the pure reflection of light in the sail (sometimes referred to as a white sail) or the pure absorption of light in the sail (sometimes referred to as a black sail) [2]. For each case, the motion of a spacecraft equipped with such a sail, in the direction of the light propagation or in the opposite direction, has also been studied [3]. In textbooks, the problem was addressed as well, usually assuming that the outgoing photons, in the case of a mirror

3 Author to whom any correspondence should be addressed. 
sail, do not change the frequency with respect to the incoming ones (non-relativistic approach).

The present problem is tackled, in most cases, by separately using the three-momentum and the energy conservation equations [4]. However, here we address it by using the fourvector formalism of special relativity [5], and we strongly advise such use. In fact, by using four-vector equations, we are treating, simultaneously and on an equal footing, both the conservation of the linear momentum and the conservation of the energy. This is indeed an obvious pedagogical advantage since linear momentum and energy are components of the same four-vector. Moreover, Lorentz transformations applied in the framework of the fourvector formalism, which is an inherently covariant one, allows one to readily obtain equivalent descriptions in any inertial reference frame [6]. This by itself illustrates the powerfulness of the formalism.

We are aware that devices such as solar sails are far from operational, or whether they can be constructed at all. Nevertheless, conceptually they can be assumed and analysed. Let us consider a perfect reflecting sail, i.e. a mirror, perpendicularly impacted by photons of momentum $p=h \nu / c$. The non-relativistic approach makes use of Newton's law, and one readily obtains

$$
\dot{N} \frac{2 h \nu}{c} \Delta t=M\left(v_{\mathrm{f}}-v_{\mathrm{i}}\right)
$$

where $\dot{N}=\mathrm{d} N / \mathrm{d} t$ is the number of photons per unit of time impacting on the sail's surface, $M$ is the mass of the system (e.g. sail plus spacecraft) and $v_{\mathrm{i}}$ and $v_{\mathrm{f}}$ are its initial and final velocities in the time interval $\Delta t$. In this description, one assumes the frequency of the incident and of the reflected photon to be the same, $\nu$, something that cannot be strictly true because the kinetic energy of the system does change, and the total energy must be conserved.

On the other hand, such description is certainly limited because it is neither covariant under Galileo transformations (because of the photons) nor under Lorentz transformations (because of the use of Newton's second law). In fact, the way to describe phenomena involving the photon-matter interactions, fully respecting the energy-momentum conservation in a covariant way, is by means of the special relativity formalism [7]. This is the main purpose of this article, using the processes of absorption and reflection of photons in a sail as concrete examples.

The paper is organised in the following way. In section 2 we analyse the mirror sail, and in section 3 we address the absorbing sail, taking into account the intrinsic thermodynamical effects. In section 4 we summarise the conclusions.

\section{The sail as a perfect mirror}

The internal energy, $E_{0}$, of a body at rest and its inertia, $\mathcal{M}(T)$, at temperature $T$, are related by $\mathcal{M}(T) c^{2}=E_{0}(T)$. This energy can be written as [5]

$$
E_{0}(T)=\sum_{k} m_{k} c^{2}-|\tilde{U}|+\mathcal{M}(0) \int_{0}^{T} \bar{c}(T) \mathrm{d} T,
$$

where $m_{k}$ is the mass of the constituent elementary particle $k$ and $|\tilde{U}|$ is the sum of all binding energies (nuclear, electrostatic, crystal, etc) and $\mathcal{M}(0)$ is the inertia of the body at zero kelvin; in the integral term, $\bar{c}(T)$ is the specific heat of the material. We shall admit that the sail is such a body, moving with respect to the light source (such as the Sun) with velocity $\pm v$ along 
the $x$ axis. Moreover, we assume that the source is at rest and emits in a single frequency (monochromatic approximation [8]).

The sail reflects the incident radiation and, during the process, its temperature is constant, hence there is no internal energy variation and the inertia remains constant, $\mathcal{M}(T)=\mathcal{M}$.

\subsection{Sail travelling in the direction of the light propagation}

Let us denote by $E^{\mu}$ the energy-momentum four-vector assigned to the sail, with energy $E=\gamma \mathcal{M} c^{2}$ and linear momentum $p=\gamma \mathcal{M} v$ :

$$
E^{\mu}=\left(\begin{array}{c}
c \gamma \mathcal{M} v \\
0 \\
0 \\
\gamma \mathcal{M} c^{2}
\end{array}\right)
$$

where $\gamma=\left(1-\beta^{2}\right)^{-1 / 2}$ and $\beta=v / c$, with $v$ the velocity of the sail.

For $N$ photons ( $N$ is an arbitrary integer number) with the same frequency, $\nu$, travelling in the positive $x$ direction, the energy-momentum four-vector is [9]

$$
E_{\mathrm{ph}}^{\mu}=\left(\begin{array}{c}
N h \nu \\
0 \\
0 \\
N h \nu
\end{array}\right)
$$

(the first component becomes $-N h \nu$ if the light propagates in the opposite direction). The energy-momentum conservation for the process of reflection of light on the sail's mirror surface means that the sum of the variations of the energy-momentum four-vectors for the sail and for the radiation must vanish. In infinitesimal form:

$$
\mathrm{d} E^{\mu}+\mathrm{d} E_{\mathrm{ph}}^{\mu}=0 \text {. }
$$

For an infinitesimal time interval, $\mathrm{d} t$, equation (5) explicitly reads

$$
\left(\begin{array}{c}
c \mathcal{M d}(\gamma v) \\
0 \\
0 \\
\mathcal{M d}\left(\gamma c^{2}\right)
\end{array}\right)+\left(\begin{array}{c}
\dot{N} h\left(-\nu_{\mathrm{r}}-\nu\right) \mathrm{d} t \\
0 \\
0 \\
\dot{N} h\left(\nu_{\mathrm{r}}-\nu\right) \mathrm{d} t
\end{array}\right)=0
$$

where $\nu_{\mathrm{r}}$ is the frequency of the photon reflected by the mirror sail, whose momentum is $-h \nu_{\mathrm{r}} / c$ when $v$ is the velocity of the sail. Equation (6) can be written in the following form [10]:

$$
\left\{\begin{array}{l}
\mathcal{M} \mathrm{d}(\gamma \nu)=\dot{N} h c^{-1}\left(\nu+\nu_{\mathrm{r}}\right) \mathrm{d} t \\
\mathcal{M} \mathrm{d}\left(\gamma c^{2}\right)=\dot{N} h\left(\nu-\nu_{\mathrm{r}}\right) \mathrm{d} t
\end{array}\right.
$$

The increment of the sail linear momentum is due to the impulse imparted by the photons (first equation). The second equation states that the increase of the kinetic energy of the sail is accompanied by a decrease of the frequency of the light $\left(\nu_{\mathrm{r}}<\nu\right)$. Now, we can use the following relation (Hamilton equation) [5] valid for a constant inertia body,

$$
v \mathrm{~d}(\gamma v)=\mathrm{d}\left(\gamma c^{2}\right)
$$

in the first of equations (7), to obtain

$$
\mathcal{M} \mathrm{d}\left(\gamma c^{2}\right)=\dot{N} c^{-1} h\left(\nu+\nu_{\mathrm{r}}\right) v \mathrm{~d} t
$$


From the second equation (7) combined with (9) one readily arrives at the following relation between the initial and final frequencies [3]:

$$
\nu_{\mathrm{r}}=\nu \frac{1-\beta}{1+\beta} \text {. }
$$

This frequency $\nu_{\mathrm{r}}$ depends on the velocity of the sail, $v$. The relative frequency shift due to the reflection is

$$
\frac{\nu_{\mathrm{r}}-\nu}{\nu}=-\frac{2 \beta}{1+\beta} .
$$

It is worthwhile to examine the ultra-relativistic and the non-relativist limiting cases:

$$
\lim _{\beta \rightarrow 1} \nu_{\mathrm{r}}=0
$$

i.e. the sail absorbs almost the total photon energy for high velocities; and

$$
\lim _{\beta \rightarrow 0} \nu_{\mathrm{r}}=\nu
$$

i.e. for low velocities there is almost no frequency variation. Inserting the result (13) back in (9) one arrives, in this non-relativistic limit, to equation (1). In the same classical regime, we can still obtain, from equation (11) [4],

$$
\frac{\Delta \nu}{\nu}=-2 \beta
$$

It is interesting to evaluate the fractions on the incident linear momentum and energy that are taken by the sail. As far as the linear momentum is concerned, we may define the ratio of the sail linear momentum variation with respect to the momentum ported by the incident photons as

$$
r_{p}=\frac{\mathcal{M d}(\gamma v)}{\dot{N} h \nu c^{-1} \mathrm{~d} t} .
$$

Using the first of equations (7) and (10), one readily concludes that

$$
r_{p}=\frac{2}{1+\beta}
$$

Similarly, the fraction of the incident radiation energy that goes into kinetic energy of the sail is

$$
r_{K}=\frac{\mathcal{M} c^{2} \mathrm{~d}(\gamma-1)}{\dot{N} h \nu \mathrm{d} t}
$$

Using the second equations (7) and (10), one now concludes that [2]

$$
r_{K}=\frac{2 \beta}{1+\beta}
$$

It is also instructive to calculate both the ultra-relativistic and the non-relativistic limits of $r_{p}$ and $r_{K}$. For the former case

$$
\lim _{\beta \rightarrow 1} r_{p}=1, \quad \lim _{\beta \rightarrow 1} r_{K}=1
$$

i.e. for sail velocities close to $c$, the incident energy practically goes to kinetic energy of the sail because $E \approx c p$ in this limit. On the other hand, for the latter case 


$$
\lim _{\beta \rightarrow 0} r_{p}=2, \quad \lim _{\beta \rightarrow 0} r_{K}=2 \beta \rightarrow 0,
$$

i.e. for small sail velocities the change in its kinetic energy is very small.

\subsection{Sail moving towards the light source}

For this case, the treatment is similar and the final results can be obtained from the previous expressions by replacing $v \rightarrow-v$ (equivalently, $\beta \rightarrow-\beta$ ). One obtains [3] (note that $0<\beta<1)$

$$
\nu_{\mathrm{r}}=\nu \frac{1+\beta}{1-\beta}
$$

i.e. the frequency increases. One has the following limiting cases:

$$
\lim _{\beta \rightarrow 1} \nu_{\mathrm{r}}=\infty, \quad \lim _{\beta \rightarrow 0} \nu_{\mathrm{r}}=\nu
$$

If the sail moves with a velocity close to $c$, the frequency of the reflected photon considerably increases while the sail decelerates. The limiting cases for $r_{K}$ are now

$$
\lim _{\beta \rightarrow 1} r_{K}=-\infty, \quad \lim _{\beta \rightarrow 0} r_{K}=-\beta \rightarrow 0 .
$$

\subsection{Lorentz transformation}

Let us define the reference frame $\mathrm{S}$ as the one where the light source is at rest. Reference frame $S^{\prime}$ moves with respect to $S$ with velocity $V$ along the $x$ axis (standard configuration). According to the principle of relativity, the observer in $S^{\prime}$ writes down the energy-momentum conservation describing the same process as

$$
\left(\begin{array}{c}
c \mathcal{M d}\left(v^{\prime} \gamma^{\prime}\right) \\
0 \\
0 \\
\mathcal{M d}\left(\gamma^{\prime} c^{2}\right)
\end{array}\right)=\left(\begin{array}{c}
\mathrm{d} N h \nu^{\prime} \\
0 \\
0 \\
\mathrm{~d} N h \nu^{\prime}
\end{array}\right)-\left(\begin{array}{c}
-\mathrm{d} N h \nu_{\mathrm{r}}^{\prime} \\
0 \\
0 \\
\mathrm{~d} N h \nu_{\mathrm{r}}^{\prime}
\end{array}\right)
$$

where $v^{\prime}, \nu^{\prime}$, and $\nu_{r}^{\prime}$ are the velocity the frequencies as they are measured in $\mathrm{S}^{\prime}, \gamma^{\prime}=\gamma\left(v^{\prime}\right)$ and $\mathrm{d} N$ is the number of photons involved in the process.

We stress that this description in $\mathrm{S}^{\prime}$ could be obtained from the description in $\mathrm{S}$ by means of the Lorentz transformation, described by $\mathcal{L}_{\nu}^{\mu}(V)$, applied to (5) [5], namely

$$
\mathcal{L}_{\nu}^{\mu}(V)\left[\mathrm{d} E^{\nu}+\mathrm{d} E_{\mathrm{ph}}^{\nu}=0\right] \Longrightarrow \mathrm{d} E^{\prime \mu}+\mathrm{d} E_{\mathrm{ph}}^{\prime \mu}=0 .
$$

Here

$$
\mathcal{L}_{\nu}^{\mu}(V)=\left(\begin{array}{cccc}
\gamma_{V} & 0 & 0 & -\beta_{V} \gamma_{V} \\
0 & 1 & 0 & 0 \\
0 & 0 & 1 & 0 \\
-\beta_{V} \gamma_{V} & 0 & 0 & \gamma_{V}
\end{array}\right)
$$

with $\gamma_{V}=\gamma(V)$ and $\beta_{V}=\beta(V)$. 
Indeed, it can be checked that both descriptions, in $S$ and in $S^{\prime}$ are compatible provided

$$
\beta^{\prime}=\frac{\beta-\beta_{V}}{1-\beta \beta_{V}}, \quad \nu^{\prime}=\nu\left(\frac{1-\beta_{V}}{1+\beta_{V}}\right)^{1 / 2}, \quad \nu_{\mathrm{r}}^{\prime}=\nu_{\mathrm{r}}\left(\frac{1+\beta_{V}}{1-\beta_{V}}\right)^{1 / 2},
$$

which are the transformed velocity and the relativistic Doppler shifted frequencies. In this derivation one needs equation (8) in $\mathrm{S}$ and in $\mathrm{S}^{\prime}$.

One should note that an attempt to get the description in $S^{\prime}$, starting from (1), using the transformed quantities (27) is bound to fail because that equation (1) is valid only in the nonrelativistic regime.

An advantage of the four-vector formalism, since it is intrinsically covariant, is the possibility to automatically describe the process in any inertial reference frame. For example, using equations (27) it is straightforward to obtain equation (10) in $\mathrm{S}^{\prime}$, namely $\nu_{\mathrm{r}}^{\prime}=\nu^{\prime}\left(1-\beta^{\prime}\right) /\left(1+\beta^{\prime}\right)$, as it should be; the same applies to equation (11), with $\left(\nu_{\mathrm{r}}^{\prime}-\nu^{\prime}\right) / \nu^{\prime}=-2 \beta^{\prime} /\left(1+\beta^{\prime}\right)$ in $\mathrm{S}^{\prime}$, and so on.

\section{Perfect absorbing sail}

Now we study a sail that absorbs all incident photons. For the sake of simplicity, we do not consider the radiation emission during the absorption process (assuming that it can be ignored in comparison with the other process). Because the sail heats up due to the incoming light, the inertia of the sail also varies with the temperature. The differential internal energy variation is $[5]$

$$
\mathrm{d} E_{0}=\mathcal{M}(T) \bar{c}(T) \mathrm{d} T=\mathrm{d}\left[\mathcal{M}(T) c^{2}\right]
$$

and then $\mathrm{d} \mathcal{M}=c^{-2} \mathcal{M}(T) \bar{c}(T) \mathrm{d} T$. The factor multiplying $\mathrm{d} T$ is very small, meaning that the inertia variation is tiny, even if the temperature variation in sizeable. Therefore, we could take $\mathcal{M}$ to be constant, as we did in section 2 . However, it is worthwhile, conceptually, to take into account the temperature dependence of the inertia, and we shall see how the relativistic formalism deals with this possibility.

\subsection{Sail travelling in the direction of the light propagation}

There are no outgoing photons and the energy-momentum four-vectors for the sail and the photons are given by (3) (now with $\mathcal{M}(T)$ a temperature dependent function) and (4), respectively.

The energy-momentum conservation, equation (5), yields, for an infinitesimal time interval, $\mathrm{d} t$, the following system of coupled equations:

$$
\left\{\begin{array}{l}
\mathrm{d}[\mathcal{M}(T) \gamma v]=\dot{N} h c^{-1} \nu \mathrm{d} t \\
\mathrm{~d}\left[\mathcal{M}(T) \gamma c^{2}\right]=\dot{N} h \nu \mathrm{d} t
\end{array}\right.
$$

where $v$ is the velocity of the sail, and we note that we allow $\mathcal{M}$ to vary during the process. However, as far as the 'mechanical' description is concerned, the inertia can be considered constant, equal to $\mathcal{M}$. Then, we can write the first of equations (29) as $\mathcal{M d}(\gamma v) \approx \dot{N} h c^{-1} \nu \mathrm{d} t$, and use equation (8) to obtain

$$
\mathcal{M d}(\gamma-1) c^{2} \approx \dot{N} h c^{-1} \nu v \mathrm{~d} t
$$

In the present case, the linear momentum of the incoming photon is completely transferred to the sail, hence the ratio $r_{p}$, defined in the previous section, is always $r_{p}=1$. The 
energy increment of the sail can be written as

$$
\mathrm{d} E=\mathrm{d} N h \nu=\mathrm{d}\left[\mathcal{M}(T)(\gamma-1) c^{2}\right]+\mathrm{d}\left[\mathcal{M}(T) c^{2}\right]=\mathrm{d} K+\mathrm{d} E_{0}
$$

i.e. one part is kinetic energy increase of the sail and the other part is internal energy increase. For the 'mechanical part', we use the approximate equation (30) to write

$$
\mathrm{d} K \approx \mathcal{M}(T) \mathrm{d}\left[(\gamma-1) c^{2}\right] \approx \mathrm{d} N h \nu \beta .
$$

For the 'thermodynamical part' it is conceptually relevant to take into account the inertia variation and one has

$$
\mathrm{d} E_{0}=\mathrm{d}\left[\mathcal{M}(T) c^{2}\right] \approx \mathrm{d} N h \nu(1-\beta) .
$$

It is interesting to evaluate the fraction on the incident energy that goes into internal energy of the sail:

$$
r_{\mathrm{I}}=\frac{\mathrm{d} E_{0}}{\mathrm{~d} N h \nu}=1-\beta .
$$

The ultra-relativistic and the non-relativistic limiting cases are

$$
\lim _{\beta \rightarrow 1} r_{\mathrm{I}}=0, \quad \lim _{\beta \rightarrow 0} r_{\mathrm{I}}=1 .
$$

For low velocities, the energy goes almost completely to internal energy, whereas for velocities close to $c$ there is almost no internal energy increase.

For the sake of completeness it is also interesting to look at the ratio, $r_{K}$, of the incoming energy that goes into kinetic energy of the sail [2]:

$$
r_{K}=\frac{\mathrm{d} K}{\mathrm{~d} N h \nu} \approx \beta,
$$

with the following limiting cases:

$$
\lim _{\beta \rightarrow 1} r_{K}=1, \quad \lim _{\beta \rightarrow 0} r_{K}=0 .
$$

In the regime $v \sim c$ the incoming energy goes basically to increment the kinetic energy of the sail. The infinitesimal temperature increment, $\mathrm{d} T$, can be obtained from equations (28) and (33) and one readily obtains the following limiting cases

$$
\lim _{\beta \rightarrow 1} \mathrm{~d} T=0, \quad \lim _{\beta \rightarrow 0} \mathrm{~d} T=\frac{\mathrm{d} N h \nu}{\mathcal{M}(T) \bar{c}(T)} .
$$

Again, we stress that we could assume a constant inertia, which would slightly simplify the calculations above since, in that case, $\mathrm{d} \mathcal{M}=0$. This would be a very good approximation, indeed. However, by taking into account the temperature dependence of $\mathcal{M}(T)$, on the one hand, we could stay conceptually more accurate, and, on the other hand, we could show how the relativistic formalism deals with that situation.

\subsection{Sail moving towards the light source}

The sail now reduces its speed and the new equations can be obtained from the previous ones by changing $v \rightarrow-v$ or, equivalently, $\beta \rightarrow-\beta$. Note that, in the expressions below, $\beta>0$. For the kinetic energy variation of the sail one has

$$
\mathrm{d} K \approx-\mathrm{d} N h \nu \beta
$$


and for the internal energy variation,

$$
\mathrm{d} E_{0} \approx \mathrm{d} N h \nu(1+\beta) .
$$

As before, we can work out the infinitesimal temperature variation and one finds the following two limits:

$$
\lim _{\beta \rightarrow 1} \mathrm{~d} T=\frac{2 \mathrm{~d} N h \nu}{\mathcal{M}(T) \bar{c}(T)}, \quad \lim _{\beta \rightarrow 0} \mathrm{~d} T=\frac{\mathrm{d} N h \nu}{\mathcal{M}(T) \bar{c}(T)} .
$$

Regarding the above defined ratios, one finds [3]

$$
r_{\mathrm{I}}=1+\beta \quad \text { and } \quad r_{K}=-\beta \text {. }
$$

The ratio $r_{\mathrm{I}}$ is always larger than one, because the decrease of the kinetic energy of the sail is incorporated in the body itself as internal energy.

\subsection{Lorentz transformations}

As for the mirror sail, it is instructive to look at the processes involving the absorbing sail from the point of view of the reference frame $S^{\prime}$. The observer in $S^{\prime}$ describes the photon absorption process by means of the energy-momentum conservation as

$$
\left(\begin{array}{c}
c \mathrm{~d}\left[\gamma^{\prime} \mathcal{M}\left(T^{\prime}\right) v^{\prime}\right] \\
0 \\
0 \\
\mathrm{~d}\left[\gamma^{\prime} \mathcal{M}\left(T^{\prime}\right) c^{2}\right]
\end{array}\right)=\left(\begin{array}{ccc}
\mathrm{d} N & h \nu^{\prime} \\
0 \\
0 \\
\mathrm{~d} N & h \nu^{\prime}
\end{array}\right),
$$

where primmed quantities are the velocity, the frequency and the temperature measured in $\mathrm{S}^{\prime}$. As mentioned in section 2.3, this description in $S^{\prime}$ can be obtained by means of a Lorentz transformation applied to (5).

It can be checked that both descriptions, in $\mathrm{S}$ and in $\mathrm{S}^{\prime}$, are compatible with the usual transformations (27), supplemented with $T^{\prime}=T$ [5]. In fact, using the transformations $\nu^{\prime}=\gamma_{V}\left(1-\beta_{V}\right) \nu$ and $\gamma^{\prime}=\gamma \gamma_{V}\left(1-\beta \beta_{V}\right)$, and also equation (29), one readily obtains

$$
\mathrm{d} N h \nu-\mathrm{d}(\gamma-1) \mathcal{M}(T) c^{2}=\mathrm{d} N h \nu^{\prime}-\mathrm{d}\left(\gamma^{\prime}-1\right) \mathcal{M}(T) c^{2}
$$

for an infinitesimal process. The two observers, in $S$ and in $S^{\prime}$, measure different photon energies and different sail kinetic energy variations, but they do measure the same internal energy variation [11]. From equation (44) one concludes that

$$
\mathrm{d}\left[\mathcal{M}(T) c^{2}\right]=\mathrm{d}\left[\mathcal{M}\left(T^{\prime}\right) c^{2}\right],
$$

showing that the formalism does indeed require $T=T^{\prime}$ : the temperature is a Lorentz scalar [12], and $\mathcal{M}(T)$, at any given temperature, must lead to the same result when measured in any reference frame [5]. So we agree with those authors who also argue that temperature is invariant under Lorentz transformations [13-15], although we should note that the subject has been a matter of debate over the years.

\section{Conclusions}

In this paper we used a fully relativistic covariant formalism to deal with processes of reflection and absorption of light by a macroscopic body-the sail. The formalism shows two major advantages. Firstly, it enforces the treatment of the conservation of the linear momentum and the conservation of the energy on an equal footing, because energy and linear momentum are components of the same four-vector. Secondly, the use of a covariant 
formalism automatically incorporates the principle of relativity, meaning that going from a description in one inertial reference frame to any other inertial frame amounts to applying to the energy-momentum conservation equation a Lorentz transformation.

We considered a perfect reflecting sail and a pure absorbing sail to obtain various interesting results, most of them already obtained by other authors, and therefore they can be found dispersed in the literature. Our paper presents, in a systematic way, the results for the two types of sails, and each one in the following two cases: when the sail moves in the direction of the light propagation and when it moves towards the light source. The nonrelativistic and the ultra-relativistic limits were also thoroughly discussed.

\section{ORCID iDs}

M Fiolhais (ib https://orcid.org/0000-0002-6636-9957

\section{References}

[1] Serway R A and Jewett J V Jr 2002 Principles of Physics. A Calculus-Based Text 3rd edn (Orlando, FL: Harcourt College Publishers) pp 914-6

[2] Coulter B L and Adler C G 1982 Radiant energy transfer Am. J. Phys. 50 335-7

[3] Singh S 1983 Note on radiant energy transfer Am. J. Phys. 511050

[4] Hirsch W and Kobrak M 2002 Doppler shift and energy transfer to a solar sail Phys. Educ. 37 $422-3$

[5] Güémez J, Fiolhais M and Fernández L A 2016 Relativistic mechanical-thermodynamical formalism-description of inelastic collisions Eur. J. Phys. 37015602

[6] Güémez J 2010 An undergraduate exercise in the first law of relativistic thermodynamics Eur. J. Phys. 31 1209-32

[7] Güémez J, Fiolhais M and Fernández L A 2016 The principle of relativity and the de Broglie relation Am. J. Phys. 84 443-7

[8] Shanks D 1956 Monochromatic approximation of blackbody radiation Am. J. Phys. 24 244-6

[9] Laufer G 1983 Work and heat in the light of (thermal and laser) light Am. J. Phys. 51 42-3

[10] Kipping D 2017 Relativistic light sails The Astronomical Journal 153277

[11] Bartell L S 2001 Apparent paradoxes and instructive puzzles in physical chemistry J. Chem. Educ. 78 1067-9

[12] Yuen C K 1970 Lorentz transformation of thermodynamics quantities Am. J. Phys. 38 246-52

[13] Landsberg P T and Matsas G E A 2004 The impossibility of a universal relativistic temperature transformation Physica A 340 92-4

[14] Landsberg P T and Matsas G E A 1996 Laying the ghost of the relativistic temperature transformation Phys. Lett. A 223 401-3

[15] Costa S S and Matsas G E A 1995 Temperature and relativity Phys. Lett. A 209 155-9 